\title{
An efficient hybrid classification system for high resolution remote sensor data
}

\author{
Roopesh Tamma ${ }^{1}$, T. Ch. Malleswara Rao ${ }^{2}$, G. Jaisankar ${ }^{1}$ \\ ${ }^{1}$ Dept of Geo Engineering, Andhra University, Visakhapatnam, India \\ ${ }^{2}$ School of Electronics, Sreenidhi Institute of Technology, Hyderabad, India
}

Email address:

rtamma@hotmail.com (R. Tamma),tchmr@yahoo.com (T. Ch. Malleswara Rao),jgummapu@yahoo.com(G. Jaisankar)

To cite this article:

Roopesh Tamma, T. Ch. Malleswara Rao, G. Jaisankar. An Efficient Hybrid Classification System for High Resolution Remote Sensor Data, American Journal of Remote Sensing. Vol. 1, No. 2, 2013, pp. 21-32. doi: 10.11648/j.ajrs.20130102.12

\begin{abstract}
The classification of aerial and satellite remote sensing data has become a challenging problem due to the recent advances in remote sensor technology that led to higher spatial and spectral resolutions. This research paper presents novel sensor independent algorithms and techniques for dealing with the challenges of classification of high volume remote sensor data. A fast unsupervised band reduction method is proposed to lower the dimensionality of the input image. The band reduced image is then split into two mutually disjoint pure and mixed pixel subsets by a pixel segregator built using extended mathematical morphology techniques. A novel hierarchical spectral-spatial support vector machine based classifier that adaptively includes the usage of expensive spatial information based on the pixel categorization is proposed. The final thematic map is obtained after merging the classification results of the two subsets and fixed spatial neighborhood homogenization. The accuracy, efficiency and flexibility of the developed system are demonstrated by evaluating the classification results using several hyperspectral and multispectral data sets. The obtained results demonstrate that the proposed method performs significantly better than conventional classifiers while alleviating the computational complexity involved in generating spatial information.
\end{abstract}

Keywords: Morphological Profile Operators, Spectral And Spatial Classification, Vector Ordered Statistics, Support Vector Machine (SVM), Hyperspectral, Multispectral

\section{Introduction}

Conventional pixel by pixel classifiers of remote-sensing data are based on signal modeling where statistical signalbased classification algorithms are applied on spectral information of each pixel vector and the pixel is assigned to the class that has the most similar statistical spectral characteristics. High spatial resolution data contains a lot of contextual information that can be employed to achieve higher discrimination of various spectrally similar classes. Many traditional classifiers can be enhanced by inclusion of spatial and contextual information. Consequently, joint spatial and spectral classifiers have been developed to analyze the remote sensing data better $[1,2]$. Majority of the combined spatial and spectral methodologies presented act on a single-band image [3, 4]. Linear extension of these grayscale techniques to multi- or hyperspectral data will not be meaningful due to the problem of ordering relation for different band values of the same pixel and the computation intractability introduced due to the dimensionality of the multi-valued data. Various pixel vector methods, using Markov random fields [5], multi-channel mathematical morphology [6], and textures [3, 7] for incorporating spatial and spectral data in to the classification process were proposed. The main drawback of most of these methods is the high computational time even for moderate sized data sets. Most of the current methods available for multi or hyperspectral data analysis are either pure pixel techniques, where each pixel is considered to be spectrally homogenous, or mixed pixel techniques where each pixel is treated as essentially spectrally heterogeneous. Many a times, an image set is often a combination of heterogeneous and a vast majority of homogenous pixels. Limiting the application of expensive joint spectral and spatial feature extraction to heterogeneous areas, while using pure spectral techniques on homogenous areas, will likely result in significant performance boost of the classification accu- 
racy while keeping the computation cost within an acceptable range.

Conventional statistical supervised classification methods are also hindered by limited availability of ground truth data and their inefficiency in handling high dimensional data. Over the last decade many techniques have been proposed to address the complexity and accuracy issues $[8,9,10]$. However, the available techniques are still inadequate for better utility of high resolution data. To deal with the statistical estimation ineptness in the presence of unfavorable ratio between available training samples to the features, several alternatives have been proposed to reduce the variance of the estimate for limited training samples [11, 12], but these improvements still suffer from the risk of over fitting the few available training samples and lead to a poor approximation of statistics. This stresses the need for a data model that has low sensitivity to the number of training samples. SVMs are shown to have capabilities for handling problems related to classification of RS data with robustness to dimensionality, good generalization ability, and a non-linear decision function. Support Vector Machine (SVM) is a new and very promising classification technique developed by Vapnik and his group at AT\&T Bell Labs [13]. Recently, researchers are focusing more on the study of SVM due to its useful applications in a number of areas, such as pattern recognition, multimedia, image processing and bioinformatics $[14,15,16]$.

In the context of all the aforementioned issues, the purpose of this research is to develop a set of new techniques to obtain effective, efficient and improved classification of high resolution data collected from satellite and airborne platforms. The accuracy, efficiency and flexibility of the developed methods and software are demonstrated by evaluating the classification results using several hyperspectral and multispectral data sets with a wide variety of spatial and spectral resolutions and encompassing diverse contexts such as urban, semi-urban and agricultural scenes. The results obtained when compared with the results of conventional spectral-only and spectral-spatial classifiers indicate that higher accuracies can be achieved with the use of these techniques and the proposed methods also alleviate the computational complexity involved by adaptive application of expensive spatial information.

This paper is organized in to five sections. Section II gives an overview of the proposed algorithms. Experimental data and setup are described in section III, and results are discussed in section IV. Section V concludes the paper.

\section{Proposed Method}

The flowchart of the proposed hierarchical hybrid classification scheme is show in Fig. 1. The input image data set is first subjected to an unsupervised dimensionality reduction algorithm to eliminate redundant bands. The band reduced image is then segregated into pure and mixed pixel subsets using ex- tended mathematical morphological operators. Pixels identified as pure are classified using only the spectral features, whereas classification of pixels marked as mixed employs both spectral and spatial information. The mixed pixels are then homogenized by application of an adjacency majority marker algorithm to eliminate presence of any detached classified pixels in the thematic map. The input to the classifier is an $n$ band multi- or hyper spectral image and the output will be a thematic classified map assigning each pixel of the input a unique class label. As shown, the classification system consists of the following four main phases:

- Band Reduction

- $\quad$ Pure and mixed pixel segregation

- Classification using SVMs of mixed and pure pixel subsets

- Pure pixels classified using spectral feature vectors and a simple Radial basis function (RBF) kernel.

- Mixed pixels classified using a hybrid spectral and spectral feature vector and a composite hybrid RBF kernel.

- Post-classification homogenization and merging.

Each of these phases is described in detail below.

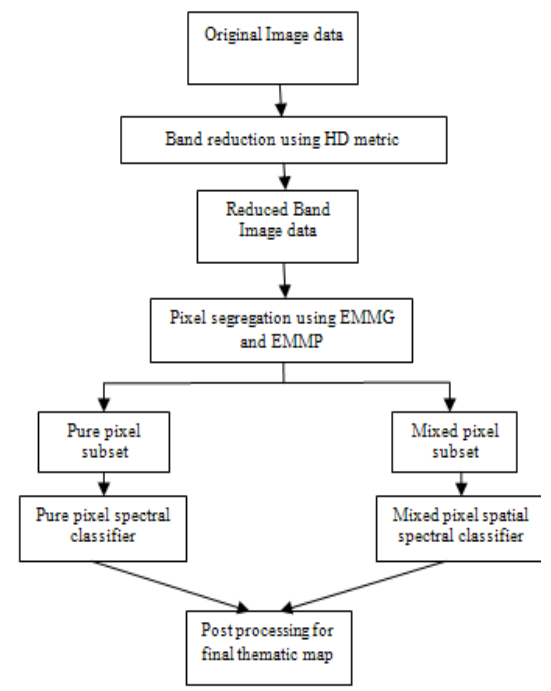

Figure 1. General architecture of the proposed classification system.

\subsection{Band Reduction}

Hyperspectral data is characterized by very high spectral resolution over a narrow range of observation channels that results in a significant number of almost redundant bands. Redundancy in data can cause convergence instability of models, increases computational resource overhead and variations due to noise in redundant data could also lead to higher discrimination inaccuracy. Thus, there is a need for a methodology that provides sufficient, but not redundant information to the classifier subsystem. In this research work, after evaluating various 
band selection methods for efficiency, a method based on Hamming distance (HD) metric is proposed. HD metric, in addition to being an unsupervised technique has the huge benefit of computationally very fast as it does not involve any higher order calculations and can be applied with the need for any object information. HD between two image band vectors $B_{\mathrm{i}}=\left\{p_{i 1}, p_{i 2} \ldots p_{i n}\right\}$ and $B_{j}=\left\{p_{j 1}, p_{j 2} \ldots p_{j n}\right\}$, where $n$ is the total number of pixels and $p_{i x}$ represents pixel value at offset $x$ in band $i$, is given by the following expression.

$$
\mathrm{HD}(\mathrm{Bi}, \mathrm{Bj})=\sum_{k=1}^{n}\left|p_{i k}-p_{j k}\right|
$$

The basic idea is that if two adjacent bands do not differ greatly then the underlying spectral-spatial property can be characterized by only one band. In general, adjacent bands that differ significantly should be retained, while similar adjacent bands can be reduced. The proposed algorithm for band reduction is presented below. In the algorithm the final Hamming distance threshold $\epsilon$ chosen will be arrived at by iteratively adjusting the threshold, starting with zero and incrementing, till the desired band count is achieved. The final band count chosen depends on the accuracy requirements and computational resources available to the user. Table 1 presents the proposed band reduction algorithm. The final band count chosen depends on the accuracy requirements and computational resources available to the user.

Table 1. Band reduction algorithm using Hamming distance metric.

Procedure BandReduction(Iband1...bandn, $\eta, E$ )

Inputs:

Orignial Image: I

Number of bands: $\eta$

Hamming distance threshold: $€$

Outputs:

Reduced band image Ibr

Begin

$\mathrm{i}=0$,

$\mathrm{Ibr}=\{\varnothing\}$,

$\mathrm{j}=\mathrm{i}+1$,

while $(\mathrm{j}<\eta)$

forall pixel vectors $\mathrm{p}$ in Bandi, and $\mathrm{pj}$ in Bandj

Hnet $=\mathrm{H}(\mathrm{Bi}, \mathrm{Bj})$,

if $($ Hnet $<C)$ then

$\mathrm{Ibr}=\operatorname{Ibr} \cup\{\mathrm{Bj}\}, \mathrm{j}=\mathrm{j}+1$,

Else

$\mathrm{i}=\mathrm{j}, \mathrm{j}=\mathrm{i}+1$,

Endif

EndWhile

Return Ibr

End

\subsection{Pure and Mixed Pixel Segregation}

The current methods available for multi or hyper spectral data analysis are inherently either pure pixel techniques, where each pixel is considered to be spectrally homogenous, or mixed pixel techniques where each pixel is treated as essentially spectrally heterogeneous. Many a times, the image set is often a combination of heterogeneous and homogenous pixels, where many sites in a scene are pure materials but many others are mixture of multiple elements. As part of this research work, it is proposed and shown that segregating the input multi or hyper spectral image data set into pure pixels and mixed pixels as a pre-classification step will help improve the accuracy and computational expense during the classification phase.

The segregation algorithm proposed is based on the observed properties of edges. Edges in any image formation model correspond to changes in discontinuities of physical properties. Generally, edges indicate overlap of two or more homogenous areas and thus, majority of the mixed pixels should lie on the edges. Features on edges have sharp boundaries. Because of the limited spatial resolution of remotely sensed images pixels on edges will contain a mixture of spectral responses from different features. Though edge detection in gray valued images is well studied, the task is less well defined in multi- or hyperspectral images. Techniques based on manifold learning [7], clustering and multivariate statistical approaches [17] were proposed to extract edges. Many of these techniques though perform satisfactorily on multispectral images, are not well suited for higher dimensional hyperspectral images. Also, these conventional algorithms like Sobel, Prewitt and Laplacian of Gaussian operator [18] are not a good fit for edge detection in images with multiple object boundaries, shadows and noise.

Filters based on mathematical morphology (MM) enable much more accurate definition of pixel neighborhoods and spatial structures in image scene than the conventional fixed closed neighborhoods at a much lower computational cost [19, 20]. The proposed pure and mixed pixel segregation algorithm uses extended mathematical morphological (EMM) operators.

Extension of mathematical morphology to multi or hyperspectral image data is not straightforward. Application of grayscale methods to each band of a multiband image is not recommended due to the existence of inherent correlation between the spectral components of various bands and a possible loss of information due to the introduction of extrinsic pixel data values [21]. The concept of vector-valued signals [22, 23] where different components of the vector contain different parts of the signal spectrum is commonly employed for handling multi-channel data by treating the data at each pixel as a vector. In order to define vector MM operations, an appropriate arrangement of vectors in the selected vector space is essential. Distance ordering where the vectors are ordered based on some distance metric between a vector and all other vectors in the sample should prove to be a valid vector ordering technique to deal with spectral feature vectors. For defining EMM operations, considering a multichannel image $f$ of $N$ dimen- 
sions as a set of pixel vectors $\left\{\mathbf{x}_{l}, \ldots, \mathbf{x}_{n}\right\}$ and a distance metric $d\left(\mathbf{x}_{i}, \mathbf{x}_{j}\right)$ to evaluate the ordering distance between two pixel vectors $\mathbf{x}_{i}$ and $\mathbf{x}_{j}$, for a set of pixel vectors within a flat structuring element $B, d_{B}(\mathbf{x})$ defines the cumulative distance between one particular pixel vector $\mathbf{x}_{i}$ and all the other pixel vectors in the spatial neighborhood defined by $B$.

$$
d_{B}(\mathbf{x})=\Sigma\left(d_{B}\left(\mathbf{x}, \mathbf{x}_{j}\right)\right) \forall \mathbf{x}_{j} \in B
$$

$d_{B}(\mathbf{x})$ can then be used to define the supremum and infimum given a set of vectors. For a given set of vectors $\mathbf{X}, d_{B}(\mathbf{x})$ is computed for every element in the set and the infimum is chosen as that $\mathbf{x}_{i}$ for which $d_{B}\left(\mathbf{x}_{i}\right)$ is the mimimum and similarly, the supremum is defined as the $\mathbf{x}_{i}$ for which $d_{B}\left(\mathbf{x}_{i}\right)$ is maximum of that set. The choice of the distance metric is an important factor in obtaining an effective ordering relation and here a $L 1$ norm was used.

Following the vector and EMM notation described in the previous chapter, the extended mathematical morphological gradient (EMMG) of an image can be obtained by computing the image difference between the dilated and eroded images. Computing the EMMG and applying a suitable threshold $\theta$ yields the edge contours. Pixels lying on these contours are most likely candidates to be marked as mixed. Additionally, further refinement of this likely-mixed pixel subset using extended mathematical morphological profiles (EMMP) is further done to identify strictly mixed pixels to achieve even greater dimensionality reduction. EMMP construction was first proposed in [6] for segmentation of high resolution images.

The algorithm for pixel segregation is given in Table 2 . The output of the algorithm will be two mutually disjoint subset image of the originally image, one subset containing all the pure pixels and the other subset made of all the pixels marked as mixed, and these two subsets are used as an input to the classification phase. In the algorithm listed below, $\left(p \circ /{ }^{\prime}\right)^{\mathrm{i}}$ denotes a geodesic dilation/erosion repeated $i$ iterations. To determine the change between EMMPs in successive iterations, HD operator is used as the metric. The iteration count parameter $k$ for the algorithm theoretically needs to be the value at which morphological idempotency is reached and, empirically it was found that a value of 5 to 7 provides good results.

Table 2. Algorithm for categorizing a pixel as pure or mixed pixel.

Procedure: PixelVectorType(I, B, $\theta$, k, refine)

Inputs:

InputImage: I, Sructuring Element: B,

EMMG threshold: $\theta$, Max \# of iterations: $k$,

Additional refinement flag: refine

Outputs:

Pixel Type: Mixed or pure for a pixel vector

Begin:

\# Compute EMMG

$\mathrm{IG}=\mathrm{EMMG}(\mathrm{I})$

For each pixel $\mathrm{p}(\mathrm{x}, \mathrm{y})$ in $\mathrm{IG}$

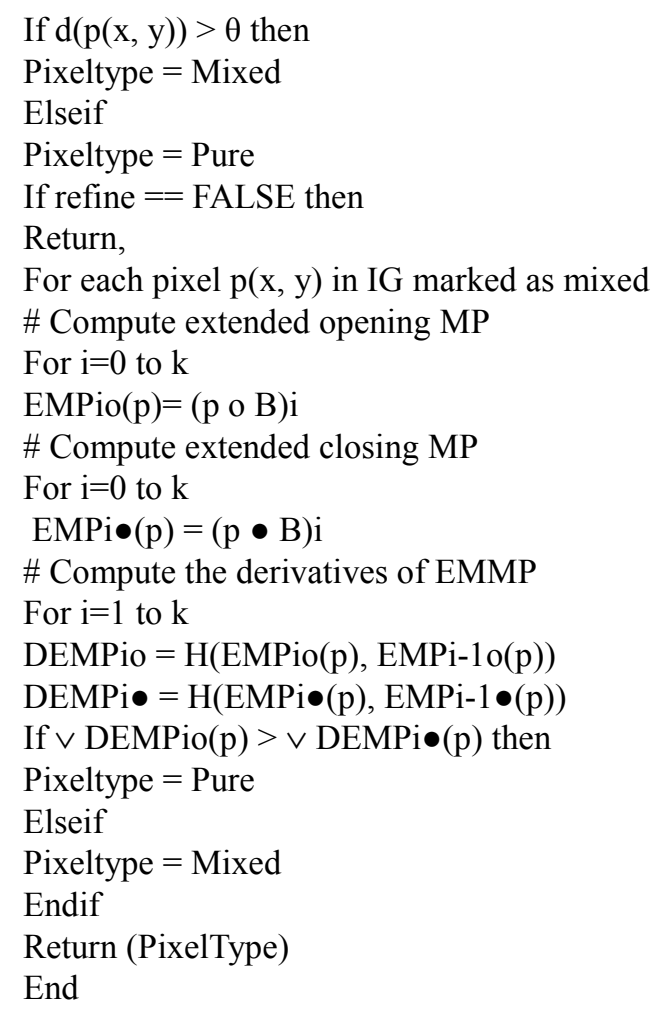

\subsection{Classification using Hybrid SVM}

SVMs with higher generalization capability, robustness to dimensionality, lower effort for model selection during learning phase and optimality of solution have proven to be more effective than the conventional parametric or non-parametric classifiers [24]. Due to all the benefits offered by SVM, in this work a hybrid classifier based on SVM method is proposed. SVM classifier design consists of choosing the appropriate feature vectors, model selection and selection of a multi-class classifier architecture.

The feature vectors chosen for this work are dynamically adapted based on the characterization of a pixel. For the subset of pixels identified as pure, the feature vector is made up of the set of the spectral values for each of the bands in the band reduced image, and for those pixels marked as mixed, for each pixel the feature vector contains the extracted spatial information measures combined with the associated spectral signatures from some or all of the bands. In this work, with an attempt to exploit the spatial information extracted using morphological profiles, an innovative way of constructing the spatial feature vector is proposed. The spatial feature vector is primarily composed of the EMMP vector with varying magnitudes of the vector. A hybrid spectral spatial feature vector is derived by the concatenation of spectral and spatial feature vectors. Model selection aims to tune the hyper-parameters of SVM classification, the penalty parameter $C$ and any kernel parameters in order to achieve the lowest test error. The choice of the kernel is a crucial issue for SVMs. Kernels introduce different nonli- 
nearities into the SVM problem and the performance of the resulting SVM will often hinge on the appropriate choice of the kernel. Though there are many kernels available in literature, the RBF kernel was chosen for this work. This kernel, unlike the linear kernel, can handle problems where the relation between class labels and attributes in not linear [25]. RBF kernel also has the additional benefit of having fewer hyperparameters and hence a lower model complexity and has only two tunable parameters: the penalty parameter $C$ and $\lambda$ the radius parameter. A multi-class is then built using one-againstone approach as the training time is much smaller compared to other approaches [26].

\subsection{Post-classification Processing}

The previous classification step produces two different classification maps, one for the pure-pixel sub-image and one for the mixed-pixel sub-image, with each pixel in the map assigned a unique class label. The post-classification processing contains merging the two disjoint classification maps to create the full image classification map, and applying contextual information regularization over the local neighborhood of all the mixed pixels.

Merging of the classification maps is a straight forward method of performing a union of the tow classification maps. The thematic map obtained from the merging operation is further refined by the application of a fixed neighborhood majority vote spatial regularization procedure for each of the mixed pixels. The area for regularization is defined by an 8-way fixed neighborhood. For each mixed pixel in the classification map, the label of the pixel is replaced by the majority vote i.e. the label with highest frequency in the chosen neighborhood area. The majority vote procedure might have to be repeated for a few iterations until stability is reached. Application of regularization reduces noise in the classification map and more homogenous regions in the final thematic map and eliminates any "salt-and-pepper" effect. The regularized classification map will be the final thematic map.

\section{Data and Experimental Setup}

For evaluating the performance of techniques developed in this work a set of hyperspectral and multispectral images from different sensors with varying spatial and spectral resolutions and covering a diverse variety of contexts and different spectral and spatial resolutions are used. Details of the data sets are listed in Table 3. Performance of the proposed algorithms was evaluated both qualitatively and quantitatively against results obtained by conventional methods. The classification system was implemented in $\mathrm{C}$ and run on a Linux $\mathrm{x} 86$ platforms. A widely used SVM library libsvm [29] was used to perform basic SVM related tasks.
Table 3. Details of data sets chosen for experimental evaluation.

\begin{tabular}{|c|c|c|c|c|c|c|c|c|}
\hline \multirow[b]{2}{*}{$\begin{array}{l}\text { Data set } \\
\text { Name }\end{array}$} & \multirow{2}{*}{\multicolumn{2}{|c|}{$\begin{array}{l}\text { Sensor Spectral } \\
\text { Type Range }\end{array}$}} & \multirow{2}{*}{\multicolumn{2}{|c|}{$\begin{array}{l}\text { \# of Image } \\
\text { BandSize in } \\
\text { s pixels }\end{array}$}} & \multirow[b]{2}{*}{ Location } & \multirow[b]{2}{*}{$\begin{array}{l}\text { Resolu- } \\
\text { tion }\end{array}$} & \multicolumn{2}{|c|}{ Groun Refer } \\
\hline & & & & & & & $\begin{array}{l}\text { d } \\
\text { Truth } \\
\text { classes }\end{array}$ & $\begin{array}{l}\text { Fig- } \\
\text { ure } \\
\text { No. }\end{array}$ \\
\hline $\begin{array}{l}\text { Indian } \\
\text { Pines }\end{array}$ & $\begin{array}{l}\text { AVI- } \\
\text { RIS }^{\mathrm{a}} \\
\text { air- } \\
\text { borne }\end{array}$ & $\begin{array}{l}10 \mathrm{~nm} \\
\text { covering } \\
\text { the wa- } \\
\text { velength } \\
\text { range } \\
\text { from } 0.4 \\
\text { to } 2.5 \mu \mathrm{m}\end{array}$ & $\begin{array}{l}n \\
- \\
220\end{array}$ & $145 \times 145$ & $\begin{array}{l}\text { Indian } \\
\text { Pines } \\
\text { site in } \\
\text { Northwes- } \\
\text { tern } \\
\text { Indiana, } \\
\text { USA [27] }\end{array}$ & $20 \mathrm{~m}$ & 16 & 2 \\
\hline $\begin{array}{l}\text { Universi } \\
\text { ty } \\
\text { of Pavia }\end{array}$ & $\begin{array}{l}\text {-ROSIS } \\
\text { air- } \\
\text { borne }\end{array}$ & $\begin{array}{l}2.9 \mathrm{~nm} \\
\text { covering } \\
\text { range } \\
\text { from } 400 \\
\text { to } 970 \mathrm{~nm}\end{array}$ & $0^{102}$ & $\begin{array}{l}1096 \times 10 \\
96\end{array}$ & $\begin{array}{l}\text { Pavia, } \\
\text { northern } \\
\text { Italy } \\
{[28]}\end{array}$ & $1.3 \mathrm{~m}$ & 9 & 3 \\
\hline $\begin{array}{l}\text { Ananta- } \\
\text { pur }\end{array}$ & $\begin{array}{l}\text { IRS }^{\mathrm{c}}- \\
1 \mathrm{~B} \\
\text { LISS }^{\mathrm{d}}-\mathrm{I}\end{array}$ & $\begin{array}{l}0.46 \\
0.86 \mu \mathrm{m}\end{array}$ & 4 & $\begin{array}{l}512 \\
512\end{array}$ & $\begin{array}{l}\text { Anantapur } \\
\text { area, } \\
\text { x Andhra } \\
\text { Pradesh, } \\
\text { India }\end{array}$ & $72.5 \mathrm{~m}$ & 15 & 4 \\
\hline Ujjain & $\begin{array}{l}\text { IRS-P6 } \\
\text { LISS- } \\
\text { IV }\end{array}$ & $\begin{array}{l}0.52 \\
0.86 \mu \mathrm{m}\end{array}$ & 3 & $\begin{array}{l}512 \\
512\end{array}$ & $\begin{array}{l}\text { Ujjain area } \\
\text { xMadhya } \\
\text { Pradesh, } \\
\text { India }\end{array}$ & $5.8 \mathrm{~m}$ & 9 & 5 \\
\hline
\end{tabular}

${ }^{a}$ Airborne Visible/Infrared Imaging Spectrometer.

${ }^{b}$ Reflective Optics System Imaging Spectrometer.

${ }^{c}$ Indian Remote Sensing Satellite.

${ }^{d}$ Linear Imaging Self Scanner.

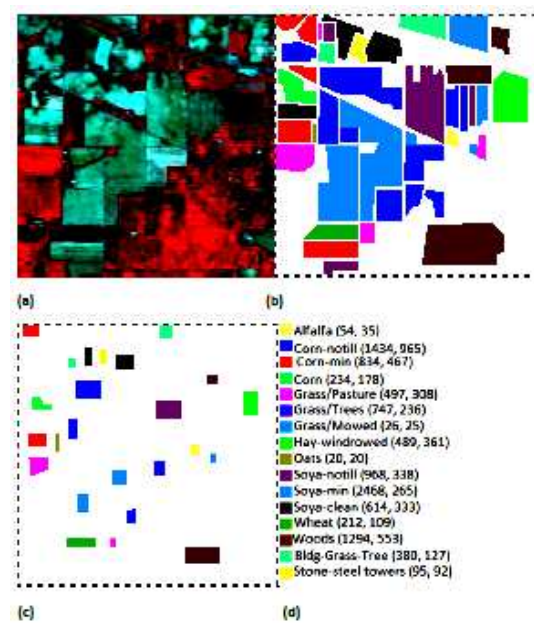

Figure 2. Indian Pines 220 band AVIRIS hyerspectral data set (AVIRIS, 1992). (a) False color composite image. (b) Training ground truth mask. (c) Test ground truth file. (d) Legend for ground truth masks with test and train sample counts shown in parenthesis as (test_sample_count, train_sample_count). 


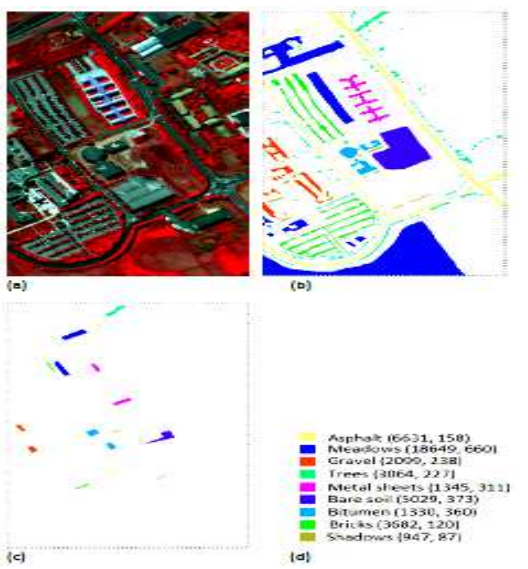

Figure 3. University of Pavia 103 band ROSIS sensor data set (Pavia, 2002). (a) False color composite image. (b) Test ground truth mask. (c) Training ground truth mask. (d) Legend for the ground truth mask with test and train sample counts given in parenthesis as (test_sample_count, train_sample_count).

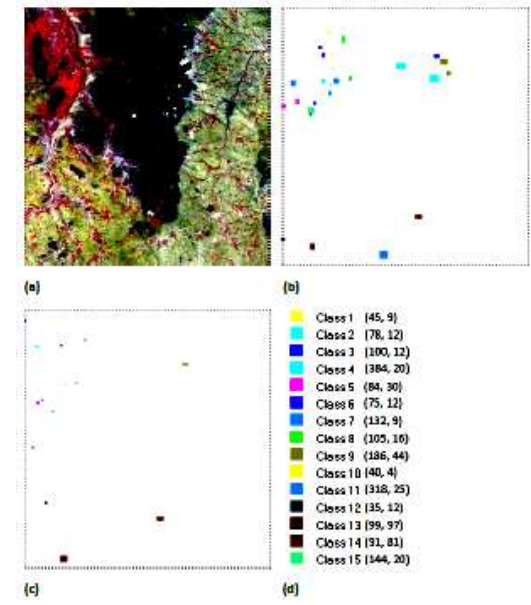

Figure 4. IRS-1B LISS-I 4 band Anantapur data set. (a) False color composite image. (b) Test ground truth mask. (c) Training ground truth mask. (d) Legend for the ground truth mask with test and train sample counts given in parenthesis as (test_sample_count, train_sample_count).

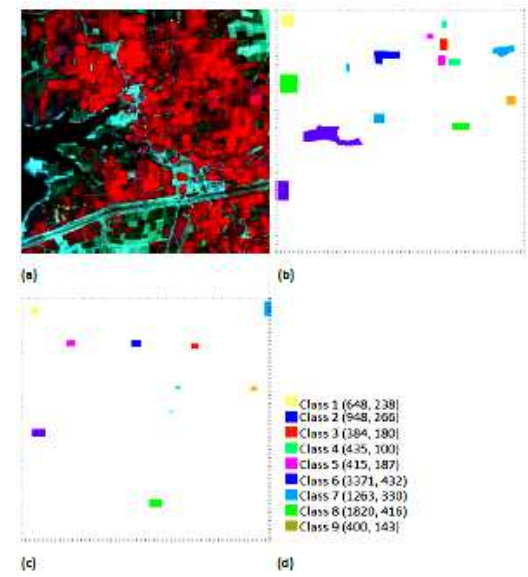

Figure 5. IRS-P6 LISS-IV 3 band Ujjain data set. (a) False color composite image. (b) Test ground truth mask. (c) Training ground truth mask. (d) Legend for the ground truth with test and train sample counts shown in parenthesis as (test_sample_count, train_sample_count).

\section{Experimental Results}

The proposed algorithms and techniques have been evaluated for accuracy and performance with the data sets presented in the previous section. The results of the experiments are discussed in this section.

\subsection{Band Reduction Algorithm Performance}

The proposed band reduction method was applied on the Indian Pines and University of Pavia data set to evaluate the performance and utility. Classification accuracy and computational complexity are the metrics used for the purpose of evaluation. The metrics are obtained on a pixel wise SVM based spectral classifier. Results of band reduction experiments on the Indian Pines data set are tabulated in Table 4. As more bands are eliminated, the accuracy gradually increases while the classification time drops steeply. This increase in accuracy as the dimensionality decreases can be explained by the Hughes phenomenon. Reduction of the bands should help filter out redundant features that add to the confusion in the decision process. Though the table does not present the memory requirements, it can be deduced that the memory requirements also drop proportional to the number of bands eliminated. Fig. 6 shows the plots that indicate the rate of band elimination and accuracy for changing values of the threshold $\epsilon$. The success of the algorithm is tightly depended on the choice of effective $\epsilon$.

Table 4. Band reduction performance for Indian Pines data set

\begin{tabular}{lllll}
\hline Epsilon $\epsilon$ & Band Count & Avg Acc & Overall Acc & $\begin{array}{l}\text { Classification Time } \\
\text { (in secs) }\end{array}$ \\
\hline 0.1 & 183 & $73.40 \%$ & $71.92 \%$ & 36.01 \\
0.4 & 168 & $74.2 \%$ & $72.99 \%$ & 27.10 \\
0.8 & 124 & $76.55 \%$ & $72.45 \%$ & 21.33 \\
1.0 & 109 & $79.15 \%$ & $72.81 \%$ & 17.43 \\
2.0 & 83 & $81.89 \%$ & $73.94 \%$ & 14.16 \\
3.0 & 62 & $82.19 \%$ & $74.58 \%$ & 13.21 \\
4.0 & 51 & $81.76 \%$ & $74.17 \%$ & 11.13 \\
5.0 & 46 & $82.34 \%$ & $75.07 \%$ & 11.11 \\
6.0 & 37 & $82.06 \%$ & $74.41 \%$ & 11.12 \\
7.0 & 33 & $53.14 \%$ & $62.13 \%$ & 11.01 \\
8.0 & 24 & $80.06 \%$ & $70.81 \%$ & 10.19 \\
9.0 & 22 & $79.16 \%$ & $71.11 \%$ & 7.17 \\
10.0 & 21 & $78.80 \%$ & $70.93 \%$ & 9.57 \\
12.0 & 19 & $76.34 \%$ & $68.17 \%$ & 8.48 \\
15.0 & 12 & $70.64 \%$ & $62.82 \%$ & 13.49 \\
20.0 & 5 & $53.10 \%$ & $51.56 \%$ & 6.39 \\
\hline
\end{tabular}




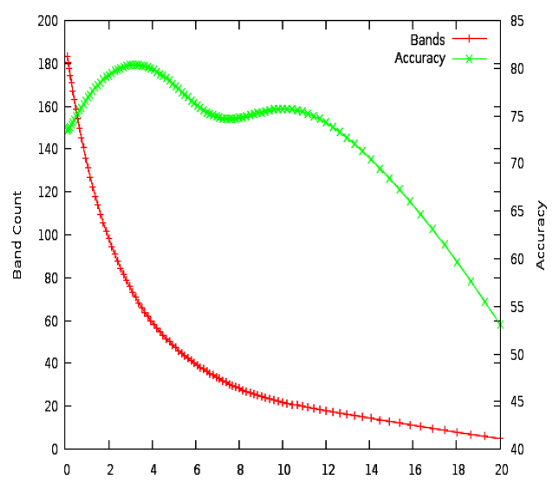

Figure 6. The effect of $\epsilon$ on band count and the relation between band count and accuracy for the Indian Pines data set.

From the curves it can be deduced that the classification performance drops steeply at $\epsilon=8.0$. After this point, the loss of features due to band elimination negatively affects the classification accuracy even though the classification time is greatly reduced. Fig. 7 plots the band count and classification time against $\epsilon$. The observed unusual increase in the classification time even with lower band count is probably due to the additional overhead involved in cross-validation phase of SVM parameter tuning. The graphs demonstrate that the best tradeoff between computation time and accuracy for this band set occurs at $\epsilon=8.0$.

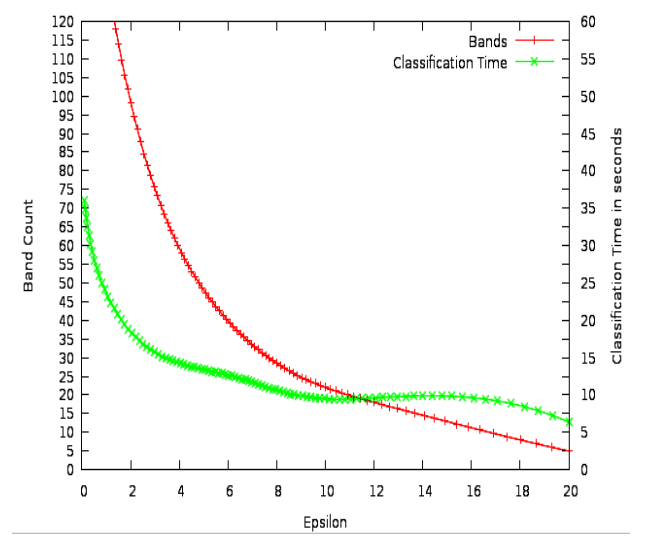

Figure 7. The effect of $\epsilon$ on band count and how the changing band count effects the classification time for the Indian Pines data set.

Results of band reduction on the University of Pavia data set are tabulated in Table 5. Figure 8 gives the rate of band elimination and accuracy for changing values of the threshold $\epsilon$ and Fig. 9 plots the band count and classification time against $\epsilon$. Similar to the results of the Indian Pines data set, the accuracy monotonically increases to a point after which it steeply drops as the number of bands eliminated increases. After the inflection point, a gradual decrease of accuracy can be observed as the classification becomes less reliable due to loss of information. The proposed method is economical and efficient as it relies on only self contained components without the need for supervised validation, expensive optimization or exhaustive search based band selection processes. The experimental results demonstrate that significant gains can be obtained even by application of coarse band reduction without noticeable loss of accuracy.

Table 5. Band reduction performance evaluation for the University of Pavia data set.

\begin{tabular}{lllll}
\hline Epsilon $\epsilon$ & Band Count & Avg Acc & Overall Acc & $\begin{array}{l}\text { Classification Time } \\
\text { (in secs) }\end{array}$ \\
\hline 0.1 & 103 & $80.59 \%$ & $74.14 \%$ & 181 \\
0.2 & 93 & $80.69 \%$ & $74.34 \%$ & 156 \\
0.4 & 70 & $80.98 \%$ & $74.78 \%$ & 124 \\
0.8 & 43 & $82.55 \%$ & $79.57 \%$ & 93 \\
1.0 & 37 & $84.71 \%$ & $81.20 \%$ & 84 \\
2.0 & 19 & $83.37 \%$ & $82.29 \%$ & 68 \\
3.0 & 13 & $79.69 \%$ & $79.29 \%$ & 62 \\
4.0 & 9 & $80.00 \%$ & $78.46 \%$ & 60 \\
5.0 & 7 & $76.38 \%$ & $78.29 \%$ & 54 \\
6.0 & 5 & $71.99 \%$ & $70.88 \%$ & 59 \\
10.0 & 3 & $69.52 \%$ & $68.86 \%$ & 73 \\
\hline
\end{tabular}

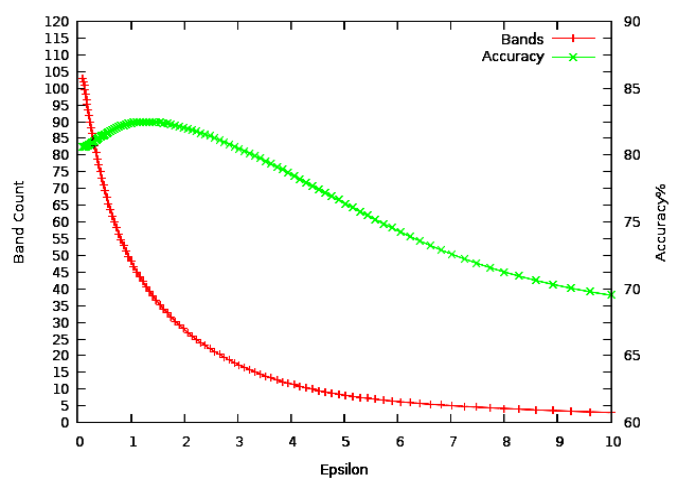

Figure 8. The effect of $\epsilon$ on band count and the relation between band count and accuracy for the University of Pavia data set.

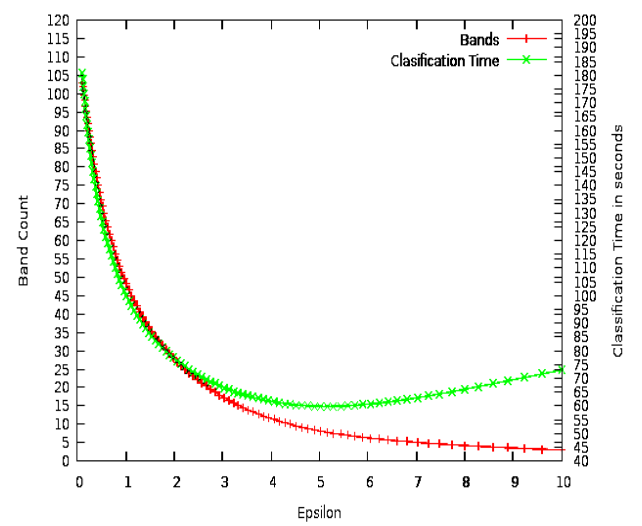

Figure 9. The effect of $\epsilon$ on band count and how the changing band count effects the classification time for the University of Pavia data set. 


\subsection{Classification Results}

The performance of the proposed classifier system is evaluated against two other conventional classifiers, the Maximum Likelihood (ML) classifier and Extraction and Classification of Homogenous Objects (ECHO) classifier [1] and the original pixel-wise SVM spectral classifier. ML classifier is well known and powerful statistical probabilistic classifier used for supervised classification. ML classification is done by using pixel-wise spectral signatures only. ECHO classifier is widely used in remote sensing community for joint spectral and spatial classification that relies on partitioning the image into statistically homogenous regions and then classifies each region as a single object. ECHO and ML classifications were performed using the widely used academic hyper- or multispectral image processing software MultiSpec [30]. Comparing results against the results produced by popular software provides for not only a complete but also a fair and bias free investigation.

Wherever possible, the same band reduced image is used as input for all the various classifiers. The accuracy is measured using the overall accuracy, average accuracy and Kappa coefficient performance measures described in a previous section. A small set of the available ground truth data was chosen as training data. Accuracies are calculated by using the trained model on the test ground truth data. Thematic maps are created for the entire data sets.

\section{Indian Pines Data Set}

The 220-band Indian Pines data set was first reduced to a 46 band image using $\epsilon=5.0$. The feature vector for spectral classification was made up of the reflectance values of each of the band. For the hybrid SVM, applying pixel segregation with $\theta=12.0$ yielded a mixed pixel map with $51.48 \%$ of the pixels being marked as mixed. SVM parameters $(C, \lambda)$ were tuned to $(32,0.5)$ using cross validation. Table 6 gives the class-specific and overall accuracies for all the classifiers applied on the Indian Pines data set. The classification maps and the mixed pixel maps are shown in Fig. 10. From the results we can see that for majority of the classes the ECHO classifier out performs both pixel-wise ML and SVM classification. For classes that are already homogenous, the SVM classifier performs better than the ML and at least as good as the ECHO classifier. This bolsters the idea that incorporating spatial information greatly enhances the classification accuracy of heterogeneous regions with a higher proportion of mixed pixels. The best global and class accuracies are obtained when applying joint spectral and spatial information using a hybrid SVM. The hybrid SVM significantly out performs both the SVM and ECHO classifier and this confirms the effectiveness of using EMMP as feature vector. From visual inspection it can be observed that misclassification occurs mostly between classes that are essentially close variants of each other (such as the corn and soya variants).
Table 6. Classification results for the Indian Pines data set.

\begin{tabular}{|c|c|c|c|c|c|c|}
\hline \multirow[b]{2}{*}{ Class } & \multirow{2}{*}{$\begin{array}{l}\text { Train } \\
\text { Samples }\end{array}$} & \multirow{2}{*}{$\begin{array}{l}\text { Test } \\
\text { Samples }\end{array}$} & \multicolumn{4}{|c|}{ Classifier Method Class Accuracies } \\
\hline & & & ML & ЕСНО & SVM & $\begin{array}{l}\text { SVM Hybr- } \\
\text { id }\end{array}$ \\
\hline $1-$ Alfalfa & 54 & 35 & 48.72 & 48.72 & 74.36 & 77.4 \\
\hline 2 - Corn no-till & 1434 & 965 & 71.39 & 83.45 & 78.18 & 81.48 \\
\hline 3 - Corn min-till & 834 & 467 & 63.01 & 75.13 & 69.64 & 83.93 \\
\hline 4 - Corn & 234 & 178 & 85.87 & 92.39 & 91.85 & 98.37 \\
\hline 5- Grass Pasture & 497 & 308 & 93.51 & 94.18 & 92.17 & 97.54 \\
\hline 6 - Grass tress & 747 & 236 & 94.69 & 96.27 & 91.68 & 97.70 \\
\hline $\begin{array}{l}7 \text { - Grass pasture } \\
\text { mowed }\end{array}$ & $e_{26}$ & 25 & 36.36 & 100 & 100 & 100 \\
\hline 8 - Hay-winrowed & 489 & 361 & 97.72 & 97.72 & 97.72 & 99.54 \\
\hline 9 - Oats & 20 & 20 & 100 & 100 & 100 & 100 \\
\hline 10 - Soyabean-notill & 1968 & 338 & 79.43 & 90.10 & 82.03 & 98.58 \\
\hline $\begin{array}{l}11 \text { - Soyabean } \min \\
\text { till }\end{array}$ & $\mathrm{n}_{2468}$ & 265 & 52.65 & 64.14 & 58.95 & 82.09 \\
\hline 12 - Soyabean clean & 614 & 333 & 85.99 & 89.89 & 87.94 & 97.70 \\
\hline 13 - Wheat & 212 & 109 & 98.15 & 98.15 & 98.77 & 99.38 \\
\hline 14 - Woods & 1294 & 553 & 95.42 & 94.21 & 93.01 & 98.38 \\
\hline $\begin{array}{l}15-\text { Bldg-Grass- } \\
\text { Drives }\end{array}$ & -380 & 127 & 73.03 & 81.52 & 61.52 & 79.70 \\
\hline $\begin{array}{l}16-\text { Stone-steel- } \\
\text { towers }\end{array}$ & -95 & 92 & 97.78 & 97.78 & 97.78 & 100 \\
\hline Overall Accuracy & & & 75.41 & 81.64 & 79.07 & 86.05 \\
\hline Average Accuracy & & & 79.61 & 83.75 & 82.34 & 90.83 \\
\hline Kappa Coefficient $\kappa$ & & & 72.25 & 80.38 & 75.33 & 86.93 \\
\hline
\end{tabular}
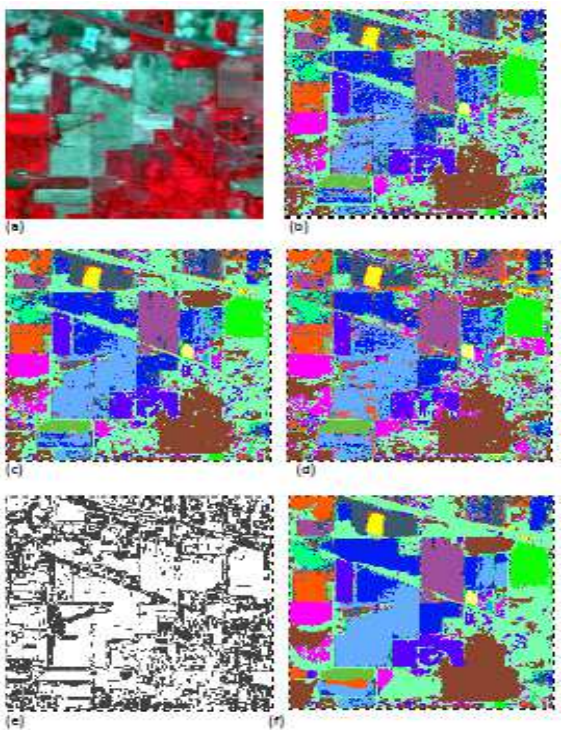

Figure 10. Indian Pines data set (a) False color composite. (b) MLC classification map. (c) ECHO classifier classification map. (d) SVM classification map. (e) Mixed pixel map (dark pixels indicate mixed pixels). (f) SVM-hybrid classification map.

University of Pavia Data Set

Pavia scene's 103 bands were reduced to a total of 37 bands 
using band reduction algorithm with $\epsilon$ set to 1.0. SVM parameters $(\mathrm{C}, \lambda)$ were tuned to $(32.0,2.0)$ using cross-validation. Table 7 reports the class and global accuracies for the University of Pavia data set. The classification thematic maps are shown in Fig. 11. Again, it is apparent from the summarized results that the classification accuracies reported by spatiospectral methods exceed spectral only methods significantly. Also the accuracy gain by the hybrid SVM compared to the ECHO classifier is higher than the Indian Pines data set. One probable reason for this is that this scene contains proportionally more straight lines with different orientations than the Indian pines scene. This demonstrates that the spatial features using EMMP provides a final classification output that is coherent in both spectral and spatial terms for a complex real-world analysis scenario.

Table 7. Classification results for the University of Pavia data set.

\begin{tabular}{|c|c|c|c|c|c|c|}
\hline \multirow{2}{*}{ Class } & \multirow{2}{*}{$\begin{array}{l}\text { Train } \\
\text { Samples }\end{array}$} & \multirow{2}{*}{$\begin{array}{l}\text { Test } \\
\text { Sam- } \\
\text { ples }\end{array}$} & \multicolumn{4}{|c|}{ Classifier Method Class Accuracies } \\
\hline & & & ML & ЕСНО & SVM & SVM Hybrid \\
\hline 1 - Roof & 412 & 3834 & 95.75 & 96.22 & 95.64 & 97.60 \\
\hline 2 - Street & 124 & 416 & 71.39 & 83.45 & 94.48 & 81.48 \\
\hline 3 - Paths & 175 & 175 & 63.01 & 75.13 & 97.71 & 83.93 \\
\hline 4 - Grass & 1928 & 313 & 85.87 & 92.39 & 99.53 & 98.37 \\
\hline 5- Trees & 405 & 212 & 93.51 & 94.18 & 79.16 & 97.54 \\
\hline 6 - Water & 1224 & 271 & 94.69 & 96.27 & 97.22 & 97.70 \\
\hline 7 - Shadow & 97 & 45 & 36.36 & 100 & 100 & 100 \\
\hline Overall Accuracy & & & 75.41 & 81.64 & 79.17 & 86.05 \\
\hline Average Accuracy & & & 79.61 & 83.75 & 85.97 & 90.83 \\
\hline $\begin{array}{ll}\text { Kappa } & \text { Coefficient } \\
\kappa\end{array}$ & & & 72.25 & 80.38 & 75.33 & 86.93 \\
\hline
\end{tabular}

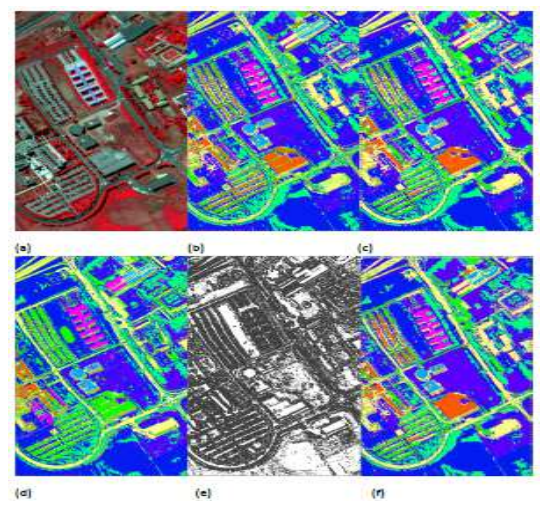

Figure 11. University of Pavia data set. (a) False color composite. (b) MLC classification map. (c) ECHO classification map. (d) SVM classification map. (e) Mixed pixel map (dark pixels indicate mixed pixels). (f) SVM-hybrid classification map.

\section{Anatapur Data Set}

For this data set no band reduction was done as the image has only four bands. With $\theta=2,59 \%$ of the pixels are identified as mixed. The higher proportion of mixed pixels is explained by the coarser resolution of the sensor and the heterogonous composition of the land area. Using cross validation the SVM parameters $(C, \lambda)$ were tuned to $(2048.0,2.0)$. Table 8 presents the classification results and the classification thematic maps are shown in Fig. 12. SVM hybrid outperforms all the other classifiers. The conventional classifiers perform badly on some classes, this can probably be explained by the fact that training regions chosen are are heterogeneous spanning across boundaries, and the possibility of incorrectly labeled training samples. ML and ECHO classifiers could not classify class 10 as the scarcity of training samples leads to covariance matrix inversion failure. SVM and SVM-hybrid classifiers were able to handle this situation and this demonstrates the robustness of the SVM based classifiers in the presence of a small training set. SVM based classifiers, unlike conventional classifiers also performed significantly better even with mixed training samples.

Table 8. Classification results for Anantaupur data set.

\begin{tabular}{|c|c|c|c|c|c|c|}
\hline \multirow[b]{2}{*}{ Class } & \multirow{2}{*}{$\begin{array}{l}\text { Test } \\
\text { Samples }\end{array}$} & \multirow{2}{*}{$\begin{array}{l}\text { Train } \\
\text { Samples }\end{array}$} & \multicolumn{4}{|c|}{ Classifier Method Class Accuracies } \\
\hline & & & $\mathrm{ML}$ & ЕСНО & SVM & $\begin{array}{l}\text { SVM Hybr- } \\
\text { id }\end{array}$ \\
\hline $1-$ Class 1 & 45 & 9 & 37.8 & 67.8 & 77.78 & 75.9 \\
\hline $2-$ Class 2 & 78 & 12 & 94.9 & 94.9 & 96.15 & 98.71 \\
\hline $3-$ Class 3 & 100 & 12 & 44.0 & 44.0 & 99.00 & 99.0 \\
\hline $4-$ Class 4 & 384 & 20 & 26.6 & 26.6 & 89.06 & 88.54 \\
\hline $5-$ Class 5 & 84 & 30 & 85.7 & 88.1 & 86.90 & 89.2 \\
\hline 6 - Class6 & 75 & 12 & 80.0 & 80.0 & 77.33 & 80.0 \\
\hline $7-$ Class 7 & 132 & 9 & 36.5 & 36.5 & 59.85 & 62.88 \\
\hline $8-$ Class 8 & 105 & 16 & 89.5 & 89.5 & 86.67 & 85.7 \\
\hline $9-$ Class 9 & 186 & 44 & 80.6 & 80.6 & 74.73 & 76.13 \\
\hline 10 - Class 10 & 40 & 4 & 0 & 0 & 37.5 & 47.5 \\
\hline 11 -Class 11 & 318 & 25 & 37.7 & 37.7 & 72.33 & 73.9 \\
\hline 12 - Class 12 & 35 & 12 & 38.0 & 38.0 & 31.43 & 37.14 \\
\hline 13 - Class 13 & 99 & 97 & 97.0 & 97.0 & 97.98 & 98.9 \\
\hline 14 - Class 14 & 91 & 81 & 89.0 & 95.6 & 89.01 & 94.5 \\
\hline 15 - Class 15 & 144 & 20 & 53.5 & 55.6 & 47.22 & 51.38 \\
\hline \multicolumn{2}{|c|}{ Overall Accuracy } & & 54.4 & 56.1 & 77.92 & 81.54 \\
\hline \multicolumn{2}{|c|}{$\begin{array}{l}\text { Average Accura- } \\
\text { cy }\end{array}$} & & 67.81 & 83.15 & 74.86 & 82.72 \\
\hline \multicolumn{2}{|c|}{$\begin{array}{l}\text { Kappa Coeffi- } \\
\text { cient } \kappa\end{array}$} & & 51.6 & 74.5 & 75.6 & 78.65 \\
\hline
\end{tabular}




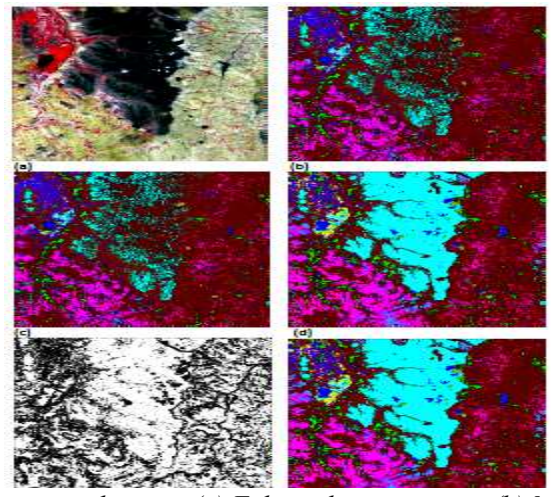

Figure 12. Anantapur data set. (a) False color composite. (b) MLC classification map. (c) ECHO classification map. (d) SVM classification map. (e) Mixed pixel map. (f) SVM-hybrid classification map.

\section{Ujjain Data Set}

This data set is made up of only three channels and hence no band reduction procedure was applied to this data set. With $\theta$ $=2.5,36.4 \%$ of the pixels are marked as mixed and the SVM parameters $(\mathrm{C}, \lambda)$ were tuned to $(32,0.5)$. Table 9 shows the classification results with various classifiers. Fig. 13 shows the classification thematic maps. Comparing the results it can be observed that SVM did only marginally better than the MLC and both methods have similar problems of misclassification of built-up land and agricultural area. SVM performs slightly worse than the MLC for certain agricultural classes, though on average the SVM performs better. This is probably due to the small deviation of the spectral data from normal distributions. The use of spatial information and homogenization of solitary pixels results in much better results for the ECHO classifier and SVM hybrid classifier.

Table 9. Classification results for Ujjain data set.

\begin{tabular}{|c|c|c|c|c|c|c|}
\hline \multirow[t]{2}{*}{ Class } & \multirow{2}{*}{$\begin{array}{l}\text { Test } \\
\text { Samples }\end{array}$} & \multirow{2}{*}{$\begin{array}{l}\text { Train } \\
\text { Samples }\end{array}$} & \multicolumn{4}{|c|}{ Classifier Method Class Accuracies } \\
\hline & & & $\overline{\text { ML }}$ & ECHO & SVM & $\begin{array}{l}\text { SVM Hybr- } \\
\text { id }\end{array}$ \\
\hline 1 -Class1 & 648 & 238 & 93.7 & 94.7 & 95.04 & 95.12 \\
\hline $2-$ Class 2 & 948 & 266 & 87.6 & 87.7 & 89.76 & 89.76 \\
\hline $3-$ Class 3 & 384 & 180 & 90.9 & 99.2 & 92.97 & 99.61 \\
\hline 4 - Class 4 & 435 & 100 & 68.0 & 77.7 & 41.72 & 81.33 \\
\hline 5 - Class 5 & 415 & 187 & 96.9 & 96.9 & 96.90 & 96.90 \\
\hline 6 - Class 6 & 3371 & 432 & 91.3 & 91.3 & 98.96 & 99.82 \\
\hline $7-$ Class 7 & 1263 & 330 & 91.7 & 91.7 & 88.04 & 92.01 \\
\hline 8 - Class8 & 1820 & 416 & 73.0 & 73.0 & 75.34 & 75.34 \\
\hline $9-$ Class 9 & 400 & 143 & 84.3 & 84.3 & 84.51 & 86.11 \\
\hline \multicolumn{2}{|c|}{ Overall Accuracy } & & 86.5 & 87.2 & 88.29 & 91.13 \\
\hline \multicolumn{2}{|c|}{$\begin{array}{l}\text { Average Accuar- } \\
\text { cy }\end{array}$} & & 86.3 & 86.9 & 88.41 & 90.67 \\
\hline \multicolumn{2}{|c|}{$\begin{array}{l}\text { Kappa Coefficient } \\
\kappa\end{array}$} & & 83.7 & 84.5 & 86.11 & 89.28 \\
\hline
\end{tabular}

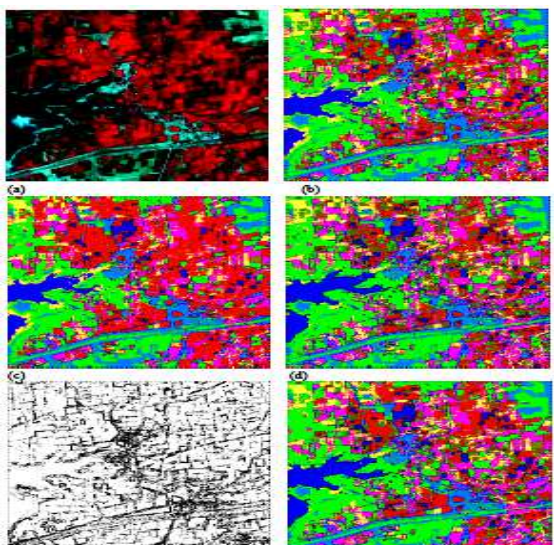

Figure 13. Ujjain data set. (a) False color composite. (b) MLC classification map. (c) ECHO classification map. (d) SVM classification map. (e) Mixed pixel map. (f) SVM-hybrid classification map.

The results demonstrate that the SVM method is technically more suitable and efficient for the classification of data sets with high dimensions as well as multispectral images. As was observed from the results of all the classifiers, it is quite apparent that the incorporating spatial information and performing homogenization using some form of inter-pixel dependency measures results in visually and qualitatively better classification maps. The hybrid SVM, by use of EMMG includes the boundary information in the yields classification maps with accurate borders. Lowering of the dimensionality by a fast and efficient band reduction method resulted in huge computational expense savings. Also, in particular, as majority of the images are composed of homogenous regions, limiting the application of spatial information only for pixels identified as mixed decreased the computational needs of the hybrid classification. On average, the proposed methodology resulted in 5$10 \%$ increase in classification accuracies without incurring any significant computational overhead.

\section{Conclusions}

This paper presented new algorithms and a system for sensor independent effective and efficient classification of hyperor multispectral images. The proposed algorithms include a fast unsupervised dimensionality reduction scheme, an elegant computationally economical algorithm for segregating pixels in the image into mixed and pure pixel categories and an adaptive hybrid classification method that selectively applies the spatial information along with spectral information. The objective of this work was the need for incorporating spatial and spectral information in multi- or hyperspectral image classification process without incurring a huge overhead in terms of computational performance. The proposed methodology succeeds in satisfying this need by taking advantage of the fact that tradeoff between accuracy and computation cost is maximized by limiting the extraction and use of spatial features 
only for the subset of pixels which mostly benefit by the use of this information in the classification process. Evaluation of the proposed techniques against existing methods has demonstrated that, on average, an increase of 5-10\% overall accuracy has been observed with less than $40 \%$ computational cost increase. Following the direction of current research for effective handling of high spatial and spectral resolution data, some of the issues that remain open and conducive for further research are identified. Application of a fast search based scheme to further optimize the proposed band reduction scheme might result in improved performance. As many of the tasks in the classification scheme lend themselves to be parallelizable both within and across modules, attempt at splitting and concurrent handling of these actions will result in reduction of overall classification time.

\section{References}

[1] Kettig, R. L., and Landgrebe D. (1976). Classification of multispectral image data by extraction and classification of homogenous objects, IEEE Transactions on Geoscience Electronics, Vol. 14, No. 1, pp. 19-26.

[2] Landgrebe D.A. (1980). The development of a spectral-spatial classifier for earth observational data. Pattern Recognition, Vol. 12 , pp. 165-175.

[3] Dobson, M. C., Pierce, L., Kellndorfer, J., and Ulbay, F. (1997). Use of SAR image texture in terrain classification. IEEE Trans. On Geosci. and Remote Sens., Vol. 3, No. 1, pp. 1180-1184.

[4] de Jong, S. M., Hornstra, T.J., and Mass, H. (2001). An integrated spatial and spectral approach to the classification of Mediterranean land cover types: the SSC method. JAG, Vol. 3, No. 2 , pp. 176-183.

[5] Tarabalka, Y., Bendiktsson, J. A., and Chanussot, J. (2010). SVM and MRF-based method for accurate classification of hyperspectral images. IEEE Trans. Geosci. Remote Sens., Vol 7, No. 4, pp. 736-740.

[6] Benediktsson, J. A., Plamson, J. A., and Sveinsson, J. (2005). Classification of hyperspectral data from urban areas based on extended morphological profiles. IEEE Transactions on Geoscience and Remote Sensing, Vol. 43, No. 3, pp. 480-491.

[7] Zhou, Y., Wu, B., Li, D., and Li, R. (2009). Edge detection on hyperspectral imagery via manifold techniques. IEEE Workshop on Hyperspectral Image and Signal Processing, WHISPERS'09, Grenoble, France.

[8] Goel, P. K., Prasher, S. O., and Patel, R. M, Landry, J. A., Bonnell, R. B., and Viau, A. A. (2003). Classification of hyperspectral data by decision trees and artificial neural networks to identify weed stress and nitrogen status of corn, Comput. Electron. Agricult., Vol. 39, pp. 67-93.

[9] Landgrebe., D. (2003). Signal Theory Methods in Multispectral Remote Sensing, John Wiley \& Sons, Inc., 2003.

[10] Camp-Valls, G., Bruzzone, L. (2005). Kernel-based methods for hyperspectral image classification, IEEE Transactions on Geoscience and Remote Sensing, vol. 43. No. 6, pp. 1351-1362.

[11] Hoffbeck, J. P. and Landgrebe, D. A (1996), Covariance matrix estimation and classification with limited training data, IEEE Transactions Pattern Anal. Machine Intelligence, Vol. 8, pp. 763-767.

[12] Tajudin, S., Landgrebe, D. A. (1999). Covariance estimation with limited training samples, IEEE Transactions on Geoscience Remote Sensing, Vol. 37, No. 4, pp. 2113-2118.

[13] Vapnik, V. N (1998). Statistical learning theory. Wiley, New York.

[14] Joachims, T. (1998). Making large scale SVM learning practical. In B. Scholkopf, C. Burges, \& A. Smola (Eds.), Advances in Kernel methods-support vector learning. MIT Press, New York.

[15] Cristianini, N., and Shawe-Taylor, J. (2000). An introduction to support vector machines and other Kernel-based learning methods. Cambridge University Press, Cambridge.

[16] Kecman, V. (2001). Learning and soft computing — support vector machines, neural networks, fuzzy logic systems. MIT Press, Cambridge.

[17] Verszkov, S., and Paclik, P. (2006). Edge detection in hyperspectral imaging - multivariate statistical approaches. Structural, Syntactic and Statistical Pattern Recognition, 4109, 551-559.

[18] Huertas, A., Medioni, G. (1986). Detection of intensity changes with subpixel accuracy using Laplacian-Gaussian masks, IEEE Transactions on Pattern Analysis and Machine Intelligence, Vol. 8, No. 5, pp. 651-664.

[19] Serra, J. (1982). Image Analysis and Mathematical Morphology, vols. 1 and 2. Academic Press, San Diego.

[20] Epifanio, I., Soille, P. (2003). Segmentation of natural landscapes using morphological texture features, Geoscience and Remote Sensing Symposium, IEEE-IGARSS Proceedings, Vol. 1, pp. 445-457.

[21] Louverdis, G., Andreadis, I., Tsalides, P. (2002). New fuzzy model for morphological color image processing, Vision, Image and Signal Processing, IEEE Proceedings, vol. 149, no. 3, pp. 129-139.

[22] Astolaa, J., Haavisto, P., \& Neuvo, Y. (1990). Vector Median Filter. Proceedings of the IEEE, Vol. 78, No. 4, pp. 679-689.

[23] Evans, A., and Liu, X. (2006). A morphological gradient approach to color edge detection. IEEE Transactions on Image Processing, Vol. 15, No. 6, pp. 1454-1463.

[24] Melgani, P., Bruzzone, L. (2004). Classification of hyperspectral remote sensing images with support vector machines, IEEE Transactions on Geoscience and Remote Sensing, vol. 42, no. 8, pp. 1778-1790.

[25] Bruzzone, L., and Prieto, D.F. (1999). A technique for the selection of kernel-function parameters in RBF neural networks for classification of remote-sensing images, IEEE Transactions on Geosicence and Remote Sensing, Vol. 37, No. 2, pp. $1179-$ 1184 . 
[26] Hsu, C., and Lin, C. (2002). A comparison of methods for multiclass support vector machines, IEEE Transactions on Neural Networks, Vol. 13, No. 2, pp. 415-425.

[27] AVIRIS (1992). AVIRIS NW Indiana's Indian Pines 1992 data set. ftp://ftp.ecn.purdue.edu/biehl/Multispec/92AV3C.lan(data set) and ftp://ftp.ecn.purdue.edu/biehl/Multispec/ ThyFiles.zip (ground truth). Accessed 24 Jan, 2011.

[28] Pavia University (2000). University of Pavia data set. www.ehu.es/uploads/e/ee/PaviaU.mat (data set) and www.ehu.es/uploads/5/50/PaviaU_gt.mat (ground truth). Ac- cessed 24 Jan, 2011.

[29] Chang, C., and Lin, C. (2001). LIBSVM: a library for support vector machines. Software available at http://www.csie. ntu.edu.tw/ cjlin/libsvm. Accessed 24 Jan 2011.

[30] Biehl, L., and Landgrebe, D. A. (2002). MultiSpec - a tool for multispectral and hyperspectral image data analysis, Computers and Geosciences, Vol. 28, No. 10, pp. 1153-1159. 